\title{
Maximum Entropy Method in Mössbauer Spectroscopy - a Problem of Magnetic Texture
}

\author{
D. SAtuŁA ${ }^{a, *}$, K. SzYmański ${ }^{a}$ And L. DobrzyŃSKI ${ }^{a, b}$ \\ ${ }^{a}$ Faculty of Physics, University of Białystok, 41 Lipowa Str., 15-424 Białystok, Poland \\ ${ }^{b}$ The Soltan Institute for Nuclear Studies, 05-400 Otwock-Świerk, Poland
}

\begin{abstract}
A reconstruction of the three dimensional distribution of the hyperfine magnetic field, isomer shift and texture parameter $z$ from the Mössbauer spectra by the maximum entropy method is presented. The method was tested on the simulated spectrum consisting of two Gaussian hyperfine field distributions with different values of the texture parameters. It is shown that proper prior has to be chosen in order to arrive at the physically meaningful results.
\end{abstract}

PACS: $76.80 .+\mathrm{y}, 02.60 .-\mathrm{x}$

\section{Introduction}

The analysis of the hyperfine parameter distribution in the Mössbauer spectroscopy have a long history. The first algorithms for the deconvolution problem was published by Varret et al. [1] with later modification by Hesse Rübartsch [2] and Window [3]. The method presented in $[1,2]$ uses a matrix formulation for reconstruction of weight function $P(x)$ ( $x$ - hyperfine parameter). In [3] an expansion of the distribution in the basis of the trigonometric functions was formulated. The problem of both methods are some mathematical artefacts which lead to unphysical oscillations in the calculated distributions from noisy Mössbauer spectra. On the other hand, deficiency of these methods consist in the necessity of postulating of some correlations between hyperfine parameters, for example linear dependence of hyperfine magnetic filed and the isomer shift. A different approach is to find $P(x)$ distribution of single parameter by the maximum entropy method presented by Brandt and Le Caer [4].

The maximum entropy method (MEM) stems from the Bayesian logic [5] was already used to analyze many spectroscopic data $[6,7,8]$. This model-free method was not frequently used to the analysis the Mössbauer spectra where presence of the distributions of hyperfine field parameters makes the spectra complicated and interpretation becomes dependent on assumptions one makes in order to get the hyperfine fields distributions. The one dimensional Mössbauer spectra contain information on the hyperfine magnetic fields $(B)$, isomer shift (IS), qudrupole splitting (QS), and orientation of the hyperfine magnetic field with respect to gamma beam

* corresponding author; e-mail: satula@alpha.uwb.edu.pl direction through magnetic texture parameter $z$ defined as:

$$
z=\frac{I_{2,5}}{I_{1,6}}=\frac{3 \sin ^{2} \theta}{\frac{9}{4}\left(1+\cos ^{2} \theta\right)},
$$

where $\theta$ is an angle between direction of gamma ray and local hyperfine magnetic field. It was shown recently that two dimensional distribution in $(B, I S)$ space [9] and three dimensional distribution in $(B, I S, Q S)[10]$ space can be reconstructed from Mössbauer spectra by the MEM technique in the first order approximation approach, where the quadrupole interaction is considered as a small parameter. The problem not discussed in $[9$, $10]$ is reconstruction of the hyperfine field distribution for not very thin samples for which there is nonlinear dependence of the intensities of Mössbauer lines and the thickness parameter. This can lead to artifacts in the reconstructed distribution. The method of extraction of cross section from the Mössbauer spectra using MEM is described in [11]. In specific problems, where a spatial arrangement of the hyperfine magnetic field depends on the field $B$, one needs to make reconstruction of the $P(B, I S$, $z$ ) distribution. The aim of this work is to test whether such reconstruction by MEM is feasible in the first order perturbation approach.

\section{Maximum entropy method}

In the application of maximum entropy method to the analysis of the Mössbauer spectra we divide whole 3 -dimensional space into pixels and the value $\rho_{j}$ denotes the probability of having given values of the chosen three parameters within j'th pixel. Because the line intensities are linear in probability, the intensities $W_{k}$ measured with uncertainties $\sigma_{k}$ at k'th velocity channel are described theoretically by a sum: 


$$
W_{k}=\sum_{j=1}^{N_{p i x}} r_{k j} \rho_{j},
$$

where $r_{k, j}$ is the transformation matrix.

As usual one is maximizing the Lagrangian

$$
L=\alpha S-\frac{1}{2} \chi^{2}
$$

where $S$ is the information entropy described as:

$$
S=-\sum_{J=1}^{N_{p i x}} \rho_{j} \ln \left(\frac{\rho_{j}}{\rho_{o j}}\right),
$$

where $\rho_{j}$ and $\rho_{o j}$ are reconstructed and initial (prior) distributions.

The final equations to solve are of type:

$$
\rho_{j}=\frac{\rho_{o j} \exp \left(-\frac{1}{2 \alpha} \frac{\partial \chi^{2}}{\partial \rho_{j}}\right)}{\sum_{j^{\prime}=1}^{N_{p i x}} \rho_{o j^{\prime}} \exp \left(-\frac{1}{2 \alpha} \frac{\partial \chi^{2}}{\partial \rho_{j^{\prime}}}\right)} .
$$

In practical applications one encounters a problem with choosing prior as this choice can influence the response of the MEM procedure.

\section{Results and discussion}

In order to test the possibility of the reconstruction of $P(B, I S, z)$ using maximum entropy method, the simulated spectrum in thin absorber approximation approach shown in Fig. 1a was used. The spectrum consists of two sextets with Voigt line shapes with hyperfine parameters, $\left(B, \sigma_{B}, I S, Q S, z\right)$, equal to $(30 \mathrm{~T}, 3 \mathrm{~T}, 0 \mathrm{~mm} / \mathrm{s}, 0 \mathrm{~mm} / \mathrm{s}$, 2) $(20 \mathrm{~T}, 4 \mathrm{~T}, 0 \mathrm{~mm} / \mathrm{s}, 0 \mathrm{~mm} / \mathrm{s}, 1)$. The integrated intensities of both sextets were assumed to be equal. Assumed hyperfine field distribution is shown in the inset of Fig. 1a. The Gaussian noise on the level of $1 \%$ of effective absorption was added to the simulated spectrum. Assumed behavior of $z(B)$ is shown in Fig. 1b. One can should point out that the reconstruction of such simulated spectrum presents one of the most difficult tasks because the lines no 1, 2 and 5, 6 of low field component are located in the positions of lines no. 2 and 5 of the high field component. One can thus expect a strong correlations between reconstructed texture parameters of both components.
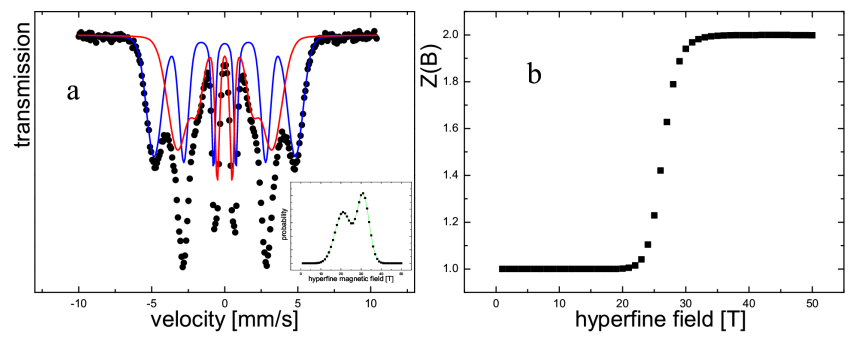

Fig. 1. a) The simulated Mössbauer spectrum and b) its $z(B)$ function. The hyperfine magnetic field distribution is shown as a inset.
At first, flat initial prior was assumed. It means that the response of MEM to the complete lack of knowledge of the distribution was tested. The results of MEM procedures are presented in Fig. 2a. As one can see the theoretical line fit well to the simulated spectrum. The reconstructed marginal distribution $P(B, I S)$ (see Fig. 2b) shows two distinct maxima located at $B \approx 20 \mathrm{~T}$ and $30 \mathrm{~T}$ what qualitatively agree with assumption done in simulated spectra. One notes that the distribution of $I S$ parameter, originally assumed to be delta-like, appeared to be rather broad. This shows a degree of uncertainty of this parameter hidden in the simulated spectrum, arising from correlations between reconstructed field $B$ and the isomer shift $I S$. On the other hand, such broad distribution of $I S$ reflects our state of knowledge on this parameter. Because of the inherent property of the Maximum Entropy Method, a flat prior used in the $I S$ reconstruction results in much broader distribution (Fig. 2b) than those obtained with Gaussian prior (Fig. 3b). The shape of the reconstructed marginal $P(B)$ distribution (Fig. 2c) is wider than the simulated one. Moreover, the low magnetic field component is moved towards lower values of $B$ (Fig. 2c). When behavior of $z(B)$ (Fig. 2d) is concerned, one notices that it is quantitatively clearly different than the one used in simulation, yet it indicates that there are different texture values for high magnetic fields than for lower. In conclusion, one can say that the use of flat prior results in good description of the Mössbauer spectrum but only in a qualitatively good reconstruction of the simulated distribution of hyperfine parameters.

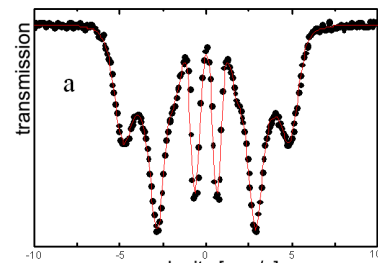

velocity $[\mathrm{mm} / \mathrm{s}]$

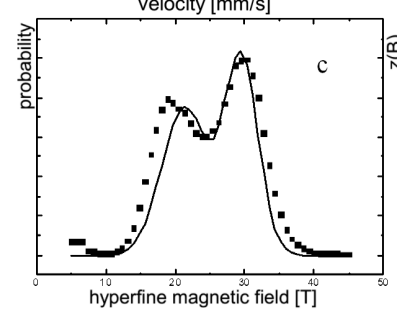

hyperfine magnetic field [T]
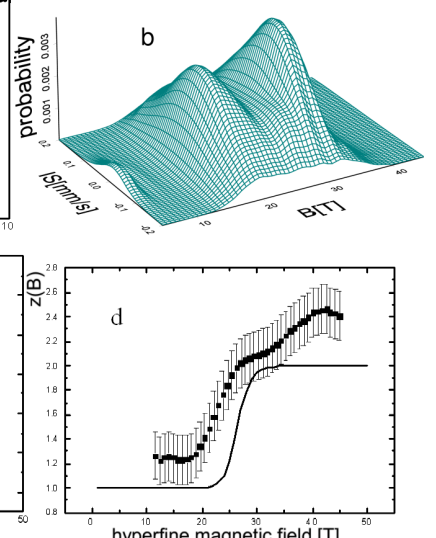

Fig. 2. a) The simulated Mössbauer spectrum (points) and fitting line (solid line); b) marginal $P(B, I S)$ distribution; c) marginal $P(B)$ distribution and d) $z(B)$ are obtained using flat prior. The solid lines in (c) and (d) represents the results of simulation.

In the second step, the prior was assumed to be a superposition of two broad Gaussian shapes (see Fig. 3a) which follow from quick inspection of the simulated Mössbauer spectrum. The position of the spectroscopic lines in the simulated spectrum shows beyond the doubts that at least two components, one at about $B \approx 30 \mathrm{~T}$ and sec- 
ond somewhere in the central part of spectra, must characterize the distribution of hyperfine parameters. The response of MEM to the chosen prior is such presented in Figs. 3b, 3c and 3d for $P(B, I S), P(B)$ and $z(B)$, respectively. As it follows from inspection of these Figures, this time the reconstructed distributions agree much better with the simulated ones than previously. $P(B)$ distribution differs from the assumed one a little only, and the behavior of $z(B)$ also fits better to the simulated one, and shows existence of two magnetic configurations of iron magnetic moments.
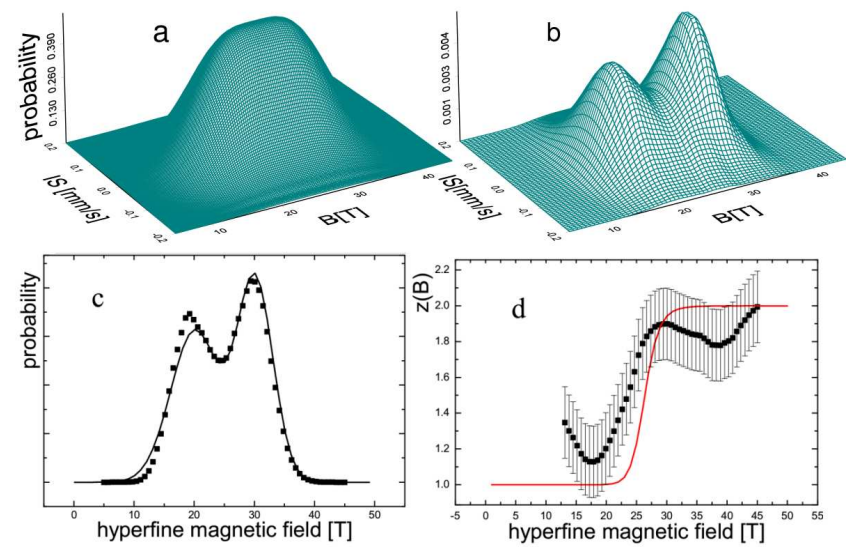

Fig. 3. a) The assumed prior distribution; b) marginal $P(B, I S)$ distribution; c) marginal $P(B)$ distribution and d) $z(B)$. The solid lines in (c) and (d) represents the results of simulation.

\section{Conclusion}

The results of our analysis show that even in very difficult case like the one analyzed in this paper, one can obtain reliable distributions of the hyperfine parameters, and obtain good indication concerning the magnetic moment configuration distribution in the considered sample. The paper shows that the use of biased prior (flat one in our case), i.e. much different from the one clearly evident from the observed spectrum, leads to distorted dis- tributions with respect to the real ones. This paper also strengthens conclusions drawn from similar analysis [11] carried out for Fe-Ni Invar alloy for which a non-collinear arrangement of magnetic moments was predicted theoretically. The results of this paper show that the MEM can be used in situations where different magnetic moment arrangements of iron are expected. As a minimum, a qualitative picture of the real $P(B, I S, z)$ distributions can be obtained and may serve as an excellent starting point to the interpretation of complicated Mössbauer spectra.

\section{Acknowledgments}

The work was partially supported by the funds allocated for scientific research for the years 2008-2011 as a research project NN202172335.

\section{References}

[1] F. Varet, A. Gérard, P. Imbert, Phys. Status Solidi B 43, 723 (1971).

[2] J. Hesse, A. Rübartsch, J. Phys. E: Sci. Instrum. 7, 526 (1974).

[3] B. Window, J. Phys. E: Sci. Instrum. 4, 401 (1971).

[4] R.A. Brand, G. Le Caer, Nucl. Instrum. Methods Phys. Res., Sect. B 34, 272 (1988).

[5] E.T. Jaynes, Probability Theory. The Logic of Science, Camridge University Press, 2003.

[6] L. Dobrzyński, X-ray Compton scattering, Oxford University Press, 2004, pp. 188-209.

[7] U. Gerhard, S. Marquardt, N. Schroeder, S. Weiss, Phys. Rev. B 58, 6877 (1998).

[8] B. Buck, V.A. Macaulary, Maximum entropy in action. Clarendon Press, Oxford 1991.

[9] L. Dobrzyński, K. Szymański, D. Satula, Nukleonika 49, S89 (2004).

[10] L. Dobrzyński, A. Holas, D. Satuła, K. Szymański, in: Bayesian Inferencje and Maximum Entropy Methods in Science and Engineering, 26 $6^{\text {th }}$ International Workshop, Ed. A. Mohammad-Djafari, American Institute of Physics, 2006.

[11] D. Satula, K. Szymański, L. Dobrzyński, J. Phys. Condens. Matter 22, 296005 (2010). 